\title{
SURVEY OF NEW SOLAR RESULTS
}

\author{
R. TOUSEY \\ E. O. Hulburt Center for Space Research, \\ U.S. Naval Research Laboratory, Washington, D.C., U.S.A.
}

\begin{abstract}
Following a brief historical review, new observations of the sun in the wavelength range 3000 to $20 \AA$ are surveyed for the period since about 1958 . Vehicles employed have been sounding rockets, the OSO (Orbiting Solar Observatories), balloons for the window 2300-1900 $\AA$ and for $\lambda>2700 \AA$, and small orbiting observatories such as Solrad, for XUV solar monitoring. Advances have been made in spectral resolution, using echelle gratings and also Fabry-Pérot interferometers. Much progress has been made towards increased spatial resolution, to obtain spectra of specific solar features and to analyse the chromosphere and corona. Methods employed include spectrographs that are stigmatic, or that have a stabilized solar image projected onto the slit; slitless objective-type spectrographs; and observations during a total eclipse. Spectra have been obtained of a solar flare, showing its form and intensity in the emission lines between 171 and $630 \AA$. From OSO 4-6 many XUV spectroheliograms and spectra have been obtained over the range 300 to $1350 \AA$, with spatial resolution $35 \times 35$ arc sec in OSO 6. Photographic XUV spectroheliograms have provided solar images with spatial resolution as great as $3 \mathrm{arcsec}$ in some cases. Although much effort has been spent to increase the accuracy of XUV intensity measurements, a great deal remains to be done before the requirements of solar physics theory are satisfied. Line identification, however, is proceeding well, although more laboratory spectroscopy is needed. Not included in the survey is the Bragg Spectrometer X-ray range below about $20 \AA$.
\end{abstract}

This Symposium deals with the subject that is basic to all of space astronomy, new techniques. It is the use of new techniques that makes possible the new observations which lead to new astrophysical theory. Dramatic proof is provided by the sequence of solar discoveries made during the first two decades of space research. The principal discoveries are listed in Table I, with the techniques involved, noted by underlining.

Space vehicles were essential, and the V 2 was the first. Two years later came the Aerobee, which is still the vehicle most used for solar research. Over the years the Aerobee has been improved in many ways. Sputnik came into being in 1957 and was soon followed by Explorer and Vanguard. All this led to the creation of NASA in 1958.

For solar research the most important device has been the biaxial pointing control, first developed by the University of Colorado in 1952 and only now being replaced by three-axis controls of at least an order of magnitude increased pointing stability.

Photography was the first technique used to record solar spectra, and it is certain to continue in use for many years to come. The first XUV spectra were recorded on fluorescence-sensitized emulsions. Great advances came through the development of Schumann-type emulsions on a film base, first SWR of Eastman Kodak, then the centrifuged emulsions of Kodak-Pathé. Solar X rays were discovered by photography, using Schumann plates manufactured by Hilger, and confirmed soon after by thermoluminescence. But photoelectric techniques took over immediately in the X-ray field, making use of the Geiger counter, and accelerating its development. The device that made possible the discovery of X-ray emission by a flare was the Rockoon, a balloon- 
TABLE I

Solar Firsts from space research

1960

1961 Photographic spectra using Al filter and grazing incidence, reaching $33 \AA$ in 1963 (NRL).

1961 Echelle spectra, high resolution - 2300-3000 $\AA$ (NRL).

1962 NASA OSO 1. Solar flux 400-171 $\AA$. Monitored by scanning spectrometer (GSFC).

1963 Matching of Zeta spectrum (UK) and high-resolution solar spectra, 171-250 $($ NRL). Followed by much Laboratory work in line identification.

1963 Bragg X-ray spectrometer - Spectra to $14 \AA$ (NRL.

1963 White-light corona without a total eclipse with rocket coronagraph (NRL).

1963 XUV spectroheliograms, using Al filter objective spectrograph technique (NRL).

1965 Off-limb XUV solar spectra with Skylark rocket, A.C.U. and limb pointer (Culham, UK).

1965 X-ray solar imagery with Wolter lens (GSFC/AS \& E).

1965 XUV spectroheliograms with channel photomultiplier from NASA OSO 2 (NRL).

1965 XUV spectra and images of flare photographed with $500 \mathrm{~km}$ Rocket (U.S.S.R.).

1965 Images of Mg II $2803 \AA$, with Šolc filter (GSFC/Stockholm).

1965 Fine spatial structure detected photoelectrically in solar H Ly-ı (NRL).

1966 XUV coronal images with heliograph (NRL).

1966 Launch of H Ly-a payload into eclipse to determine limb brightening (France).

1967 Balloon and Veronique rocket XUV experiments (France).

1967 OSO-3 Bragg crystal spectrometer (GSFC); OSO-4 XUV spectrometer/spectroheliograph (HCO); Bragg crystal spectrometer (NRL); X-Ray spectroheliometer (AS \& E).

suspended rocket that was launched by command at the moment when a flare was detected $\mathrm{H} \alpha$. First use of the eclipse technique came in 1958, when photoelectric detectors of solar X rays and XUV were flown into the eclipse path with Nike-Asp rockets launched from a naval vessel.

In the second decade many discoveries resulted from new techniques in instrumentation; photoelectric scanning monochromators. XUV spectroheliographs. 
X-ray image-forming devices, the Bragg crystal spectrometer, and so forth. At the same time there appeared new vehicles, the Skylark and Attitude Control Unit of the United Kingdom, and the French Veronique. During this period the first space observatories were placed in orbit; NRL's Solrad 1, launched in 1960, monitored the Sun's X-ray and H Ly- $\alpha$ output, inaugurating a program that has by now reached Solrad 9. The first NASA Solar Observatory (OSO) came in 1962, and three more followed, each carrying a great deal of versatile and powerful instrumentation. (For references see Tousey, 1964 and 1967.)

Prior to Sputnik, there was about one prime solar discovery per year; after this, the rate rose rapidly and has averaged about three per year. I am sure it is still increasing. The conclusion is obvious; we must continue to develop new techniques and space vehicles, expensive though this may seem to be.

I have been asked to present a survey of new solar observations in ultraviolet astronomy. I shall cover the three-year period since the XIIIth General Assembly of the IAU, and shall define the UV for today as 3000 to $20 \AA$, which includes the extreme ultraviolet (XUV) and $X$ rays down to the Bragg spectrometer region.

The region $2965 \AA$ to about $2100 \AA$ is the extension of the photospheric spectrum into the rocket ultraviolet, but now we must call it the rocket and balloon ultraviolet. Figure 1 is a spectrum covering the $\mathrm{Mg}_{\mathrm{II}} \mathrm{H}$ and $\mathrm{K}$ lines, 2802.7, 2795.5 $\AA$, made not from a rocket, but from a balloon. Depending on the state of the ozonosphere it is possible from modern balloon altitudes to reach into the UV well beyond the ground cutoff, sometimes to $2700 \AA$. There is also a second window that is accessible from

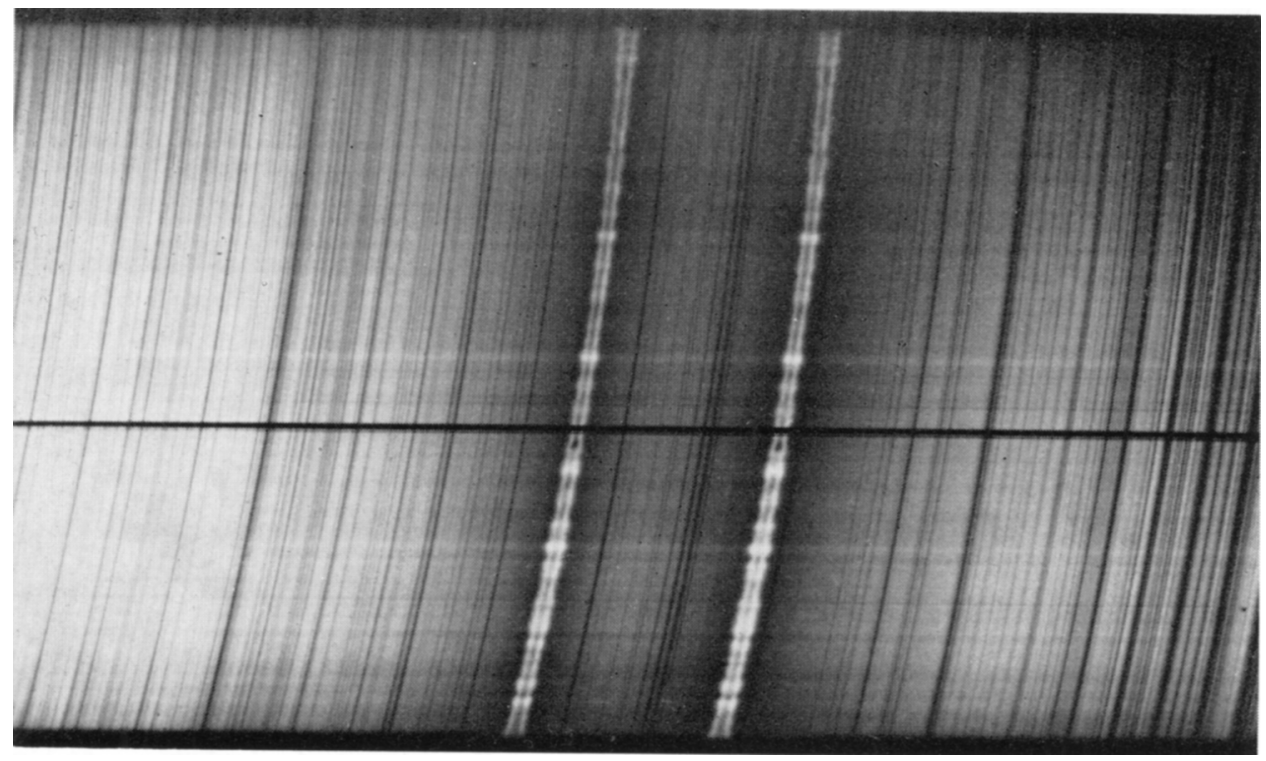

Fig. 1. A stigmatic spectrum of the sun across a solar diameter. The reversed emission lines are Mg II $2795.523 \AA$ (left) and $2802.698 \AA$. The exposure was made from a high-altitude balloon on April 30, 1969 by P. Lemaire (1969). 
balloons, 2300 to $1900 \AA$. Both of these windows have been exploited by the French.

The spectrum of Figure 1, obtained by Lemaire (1969), has very high spectral resolution, $\sim 40 \mathrm{~m} \AA$; the line profiles agree in general with the NRL echelle profiles of 1961, (Tousey et al., 1967). The new spectrum, however, is stigmatic, a great advantage over the echelle spectrum because this permits resolving spatial detail to at least 10 arc sec. I understand that from a balloon flown about one month ago Lemaire achieved $25 \mathrm{~m} \AA$ spectral and 3 arc sec spatial resolution.

The $\mathrm{MgII} \mathrm{H}$ and $\mathrm{K}$ lines and a few tens of $\AA$ ngströms nearby have also been covered with a Fabry-Pérot interferometer, the first time such a device has been flown in a rocket (Bates et al., 1969). In the most recent flight a spectral resolution of a few arc seconds was attained. Both of these balloon-borne instruments will be described in papers to follow.

In yet another way the NRL echelle spectra of 1961 and 1964 have been surpassed, to short wavelengths, as will be described in another paper at this Symposium. In Figure 2 are combined the new Culham echelle spectrum (Jones et al., 1970) and the NRL 1964 spectrum in the range where they overlap. Although differing greatly in character, the line lists agree quite well. NRL is still in process of finishing and publishing the echelle spectra and the intensity curve, which extend from about $2095 \AA$ to $3000 \AA$ with approximately $30 \mathrm{~m} \AA$ resolution, except at the short wavelength end.

Echelle gratings have been improved a great deal in the last ten or more years, and this is one reason why the $30 \mathrm{~m} \AA$ resolution Culham spectra are more intense and cleaner at the short wavelength end than those of NRL. As a result, the region of the disappearance of the photospheric spectrum is now covered very well, especially in

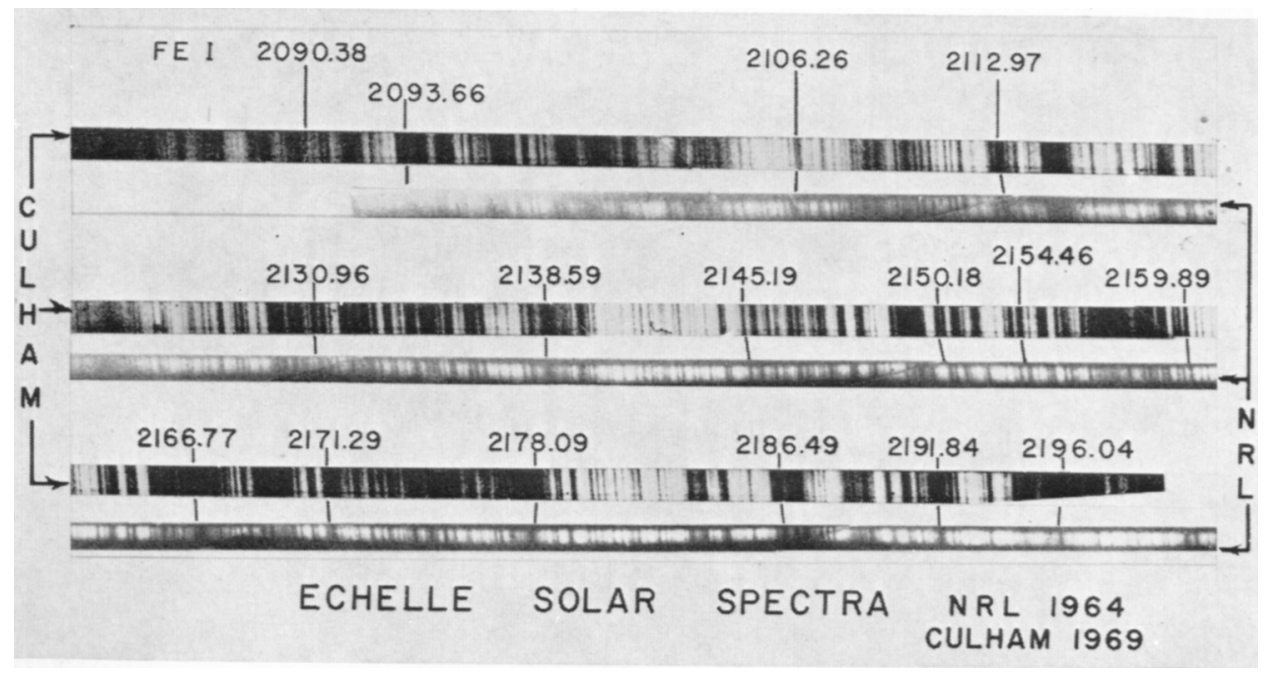

Fig. 2. Solar spectra photographed with rocket-borne echelle spectrographs, by NRL on 21 November 1964 (Tousey et al., 1967) and by the Culham Laboratory on 22 April 1969 (Jones et al., 1970). The agreement is surprisingly good, considering the great improvement in echelle gratings of the last few years. 
spectra from a later Culham flight which reach $1900 \AA$. From these high-resolution spectra it has been established beyond any question that it is the aluminum ionization edge that causes the discontinuity beyond $2087 \AA$ (Boland et al., 1970).

The region $1800-1300 \AA$ is receiving much attention of late because the radiation is a complicated mixture coming principally from the temperature minimum and the chromosphere, but with some contribution from the outer edge of the photosphere, the chromosphere-corona transition region, and the corona. Spectra covering a part of this region, obtained with three different techniques, are shown in Figure 3 . At the bottom is the February 1, 1966 NRL double-dispersion spectrum, having $0.2 \AA$ resolution (Tousey et al., 1964; Tousey, 1967b). Because the grating arrangement was stigmatic, each line shows the distribution of emission from a diametral slice across

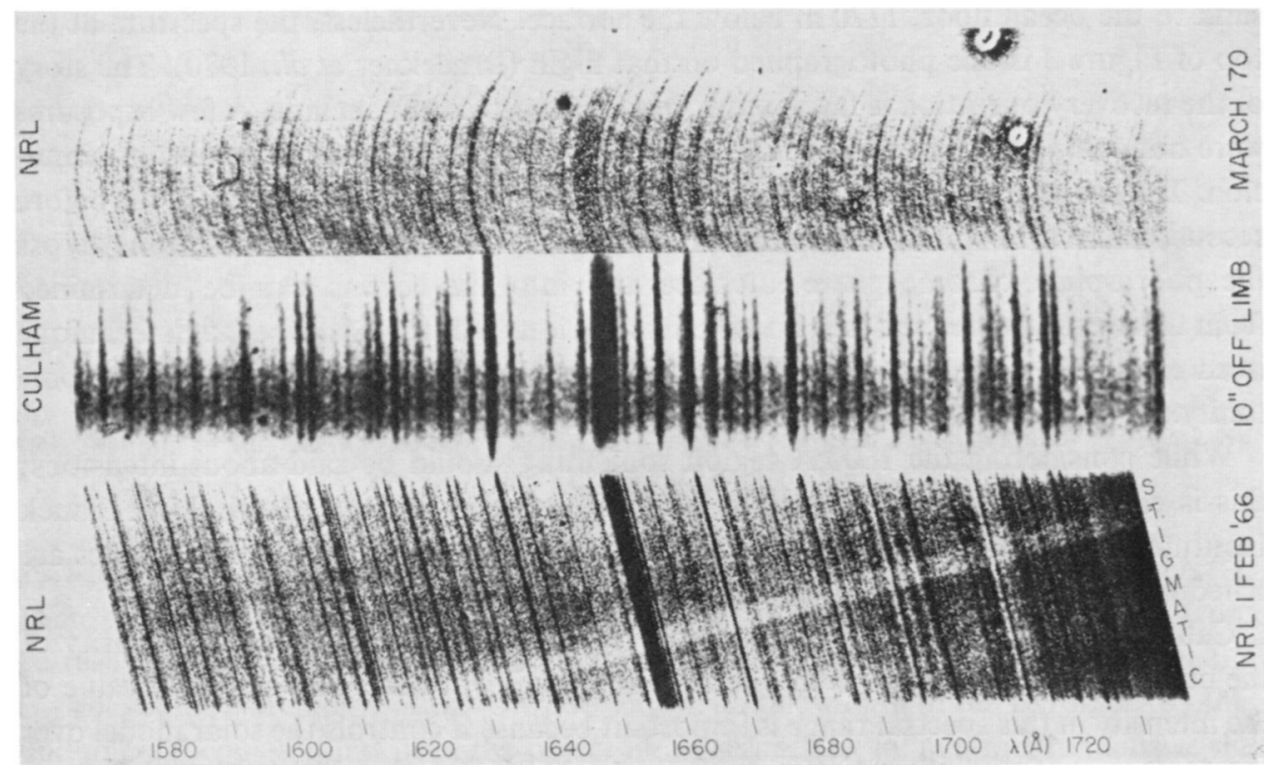

Fig. 3. XUV spectra covering the region of the sun's temperature minimum. At the bottom: NRL's stigmatic, $0.2 \AA, 10$ arc sec double-dispersion spectrum (Tousey, 1967). At the center: the 10 arc sec off-limb spectrum obtained by the Culham Laboratory on 9 April 1965 (Burton and Wilson 1965). Uppermost: A 5-s exposure flash spectrum made by NRL just before second contact on 7 March 1970, retrieved but with considerable damage from 15 days of exposure to electrolysis in sea water at a depth of $1770 \mathrm{~m}$ (Brueckner et al., 1970).

the Sun, with about 10 arc sec spatial resolution in this range. Thus the tip ends of the lines come from the chromosphere. The Culham group have obtained chromospheric spectra in a different way, by positioning the slit just above the limb, using a servomechanism to hold it steady. The center spectrum is from one of their early flights (Burton and Wilson, 1965). Many of the emission lines are greatly enhanced relative to the NRL lines where they cross the disc; they resemble more closely the tip ends of the NRL lines. but are far more suitable for photometry. 
The classical way to observe chromospheric and coronal spectra and to obtain resolution in height is by photographing the flash spectrum during a total eclipse. For the XUV this was attempted on 7 March 1970 with instruments carried in a pair of Aerobee rockets launched a few seconds apart from Wallops Island, Virginia. The one was instrumented by the Naval Research Laboratory, the other by a consortium from the Imperial College, Culham, the University of York in Toronto, and the Harvard College Observatory. The instrumentation was mainly photographic. The consortium covered the wavelength range $\approx 3000 \AA$ to $850 \AA$, the NRL $1800 \AA$ to $150 \AA$. The consortium instrument for the range 850-2150 $\AA$ produced excellent results, and established the existence of a $\mathrm{H} \mathrm{Ly}-\alpha$ corona, produced by resonance scattering by neutral hydrogen in the corona (Speer et al., 1970). The NRL instruments also operated very well, but the recovery system failed, the instrumentation crashed into the sea and sank to the ocean floor, $1770 \mathrm{~m}$ below the surface. Nevertheless, the spectrum at the top of Figure 3 is one photographed on that flight (Brueckner et al., 1970). The story of the recovery operation is fascinating, but is far too long to include. A few exposures were not completely spoiled, even though they were made with Schumann-type emulsion. The spectrum reproduced in Figure 3 is a five-second exposure made just before second contact. In spite of the damaged emulsion, the distribution of many ions across the photosphere-chromosphere interface and into the corona can be determined from the lengths of the crescent images. It is obvious that the flash spectrum technique is an extremely powerful one, and should be employed whenever there is an opportunity.

While considering the $1600 \AA$ region something should be said about intensities; this is especially apropos because in May 1968 there was held at this Max Planck Institute a symposium on calibration methods, and more papers on this subject are scheduled later in the present Symposium.

Calibration is still difficult and controversial. As a matter of fact, the intensity of the continuum near $1600 \AA$ is in question at the present time. The absolute value of the intensity in this spectral range is important because it controls the solar model over the temperature minimum where the energy transfer mechanism is changing from convection to shock waves. Figure 4 shows the latest NRL data (Widing et al., 1970), reduced from the photographic spectrum of July 27, 1966. The lowest value of the radiation temperature is $4670 \mathrm{~K}$, at $1680 \AA$. Included also are the data from the photoelectrically scanned spectrum obtained from a rocket by the Harvard College Observatory (Parkinson and Reeves, 1969); these temperatures are generally lower, with a minimum value of $4350 \mathrm{~K}$ at $1670 \AA$. It is not clear whether the explanation lies in calibration, in the separation of continuum from lines, or in the Sun itself. Additional work is required and is being undertaken. A program being set up in Japan to make measurements of this kind will be described later in this Symposium by Nishi and Suemoto.

When I feel discouraged about calibration, I think about the solar constant, a measurement that should be relatively simple, especially with the aid of space vehicles that eliminate the need for making corrections for atmospheric absorption. In Table II 


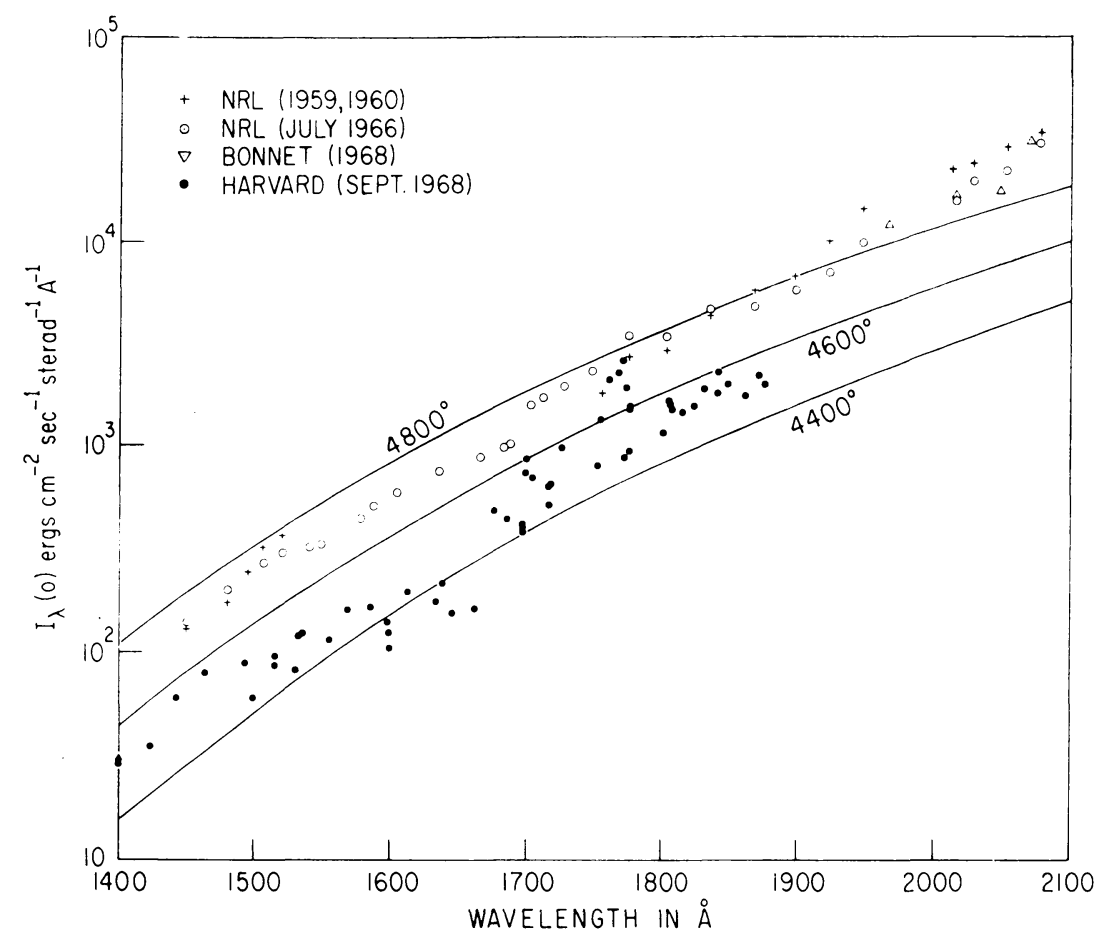

Fig. 4. The intensity of the continuum as emitted by the Sun's quiet background. The data are from NRL (Widing et al., 1970), Harvard College Observatory (Parkinson and Reeves, 1969), and a French rocket experiment (Bonnet, 1968).

are listed some values obtained in the last $15 \mathrm{yr}$, following the work of the NRL (Johnson, 1954), which led to a value $2.00 \pm 0.04 \mathrm{cal} / \mathrm{cm}^{2} / \mathrm{min}$; this was derived from the results of the Smithsonian Astrophysical Observatory but took into account the new rocket measurements of the UV correction, and new data in the IR. Recently this fundamental constant has been the object of a mass attack in an effort to increase the

TABLE II

Some values of the solar constant

Year Investigator

$\mathrm{Cal} / \mathrm{cm}^{2} / \mathrm{min}$

1954 Johnson

1956 Stair and Johnston

1958 Allen

1967 Kondratiev et al.

1968 Stair and Ellis

1968 Labs and Neckel

1968 Drummond et al.

1968 Murcray et al.

1968 Makarova and Kharitonov

1969 Arvesen, Griffin, and Pearson

1969 Thekaekara, Kruger, and Duncan
NRL

Mauna Loa-NBS

Univ. of London

U.S.S.R.

Mauna Loa

Jungfraujoch

Eppley-JPL

Denver

U.S.S.R.

Ames

GSFC
$2.00 \pm 0.04$

$2.05+0.10$

$1.99 \pm 0.02$

2.016

$1.95+0.10$

$1.958 \pm 0.02$

$1.952 \div 0.02$

1.912

$2.03 \quad 0.05$

$1.992 \quad 0.04$

$1.936 \cdot 0.04$ 
accuracy still further. Various methods have been employed, including the use of aircraft and high-altitude balloons. Nevertheless the values run all the way from 2.05 to $1.912 \mathrm{cal} \mathrm{cm}^{-2} \mathrm{~min}^{-1}$. Perhaps C. G. Abbot was right after all, the Sun is varying. Much more likely, most of the spread in values simply reflects the difficulties involved in making really accurate absolute measurements of energy, and in correcting for residual absorption effects. (References are listed under 'Solar Constant'.)

Below $1000 \AA$ normal-incidence grating instruments become slow because all materials as yet discovered have low values of reflectance. With the sun as source, long wavelength stray light is so intense, that its elimination from the XUV spectrum presents a difficult problem. In Figure 5 are shown an NRL double-dispersion photo-

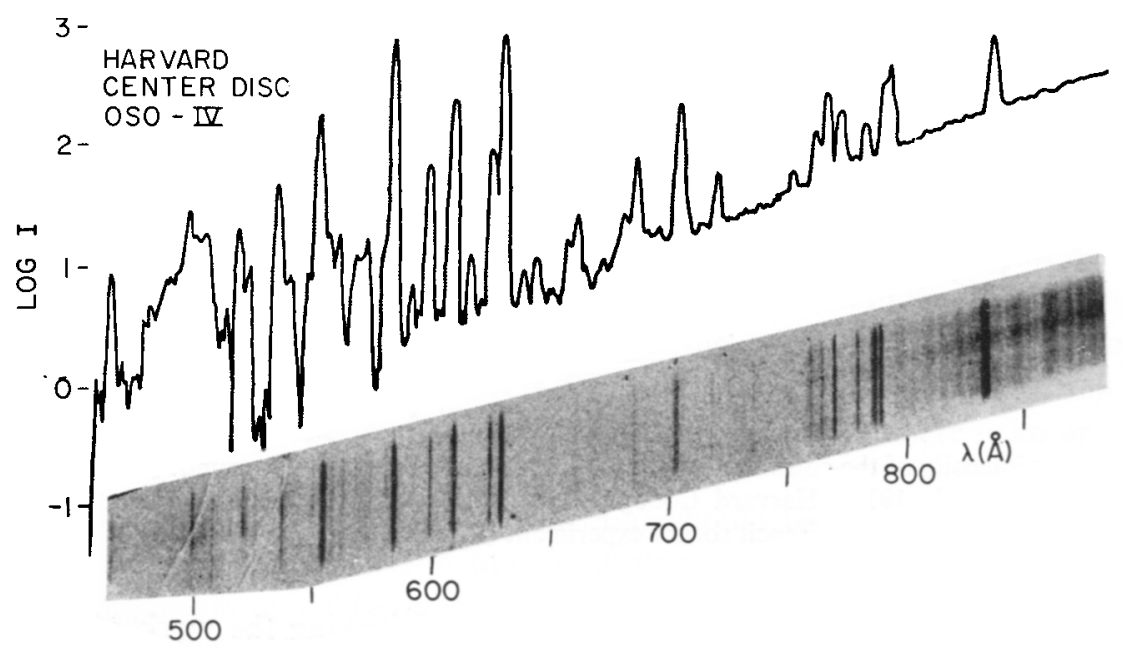

Fig. 5. The NRL double-dispersion stigmatic spectrum of 27 July 1966 compared with a spectral scan of a $1 \times 1$ arc min area at the center of the Sun's disc, made by the Harvard College Observatory photoelectric spectrometer on OSO 4.

graphic spectrum, reaching NevII, $465 \AA$ (Tousey et al., 1970), and a photoelectrically-recorded spectrum obtained by the Harvard College Observatory from OSO 4 with a scanning monochromator that received radiation from the center of the disc only (Goldberg, 1969). The spectra are similar and complementary. The spectral resolution and wavelength accuracy are greater in the photographic spectrum; the dynamic range is greater in the photoelectric spectrum and the intensity values are more precise. The absolute accuracy that can be achieved, as distinguished from precision, should be about the same, because it depends mainly on calibration.

In the OSO 6 Harvard College Observatory experiment, it was possible to point the XUV spectrometer at any desired solar feature, therefore really critical spectra of all kinds of solar features were obtained. Later in this symposium, the way this instrument was controlled to maximize the collection of new solar data will be described by Reeves. 
Grazing-incidence spectrographs and spectrometers become more useful than those using normal-incidence at about $500 \AA$. The photoelectric work of the Air Force Cambridge Research Laboratory (AFCRL) and of the Goddard Space Flight Center (GSFC) from OSO with grazing-incidence photoelectric scanning monochromators is well known (AFCRL: Hinteregger and Hall, 1969; GSFC: Neupert et al., 1969). As yet, however, solar measurements made with grazing-incidence instruments have no spatial resolution. They measure only the total emitted flux, but this they do with excellent precision and sensitivity.

Wavelengths and high spectral resolution, however, are best obtained by photography. The shortest wavelengths reached with really high resolution are shown in Figure 6, a spectrum from Culham (Freeman et al., 1970; Freeman and Jones, 1970). This includes the so-called triplets of helium-like C V, N VI, and O VII; the permitted

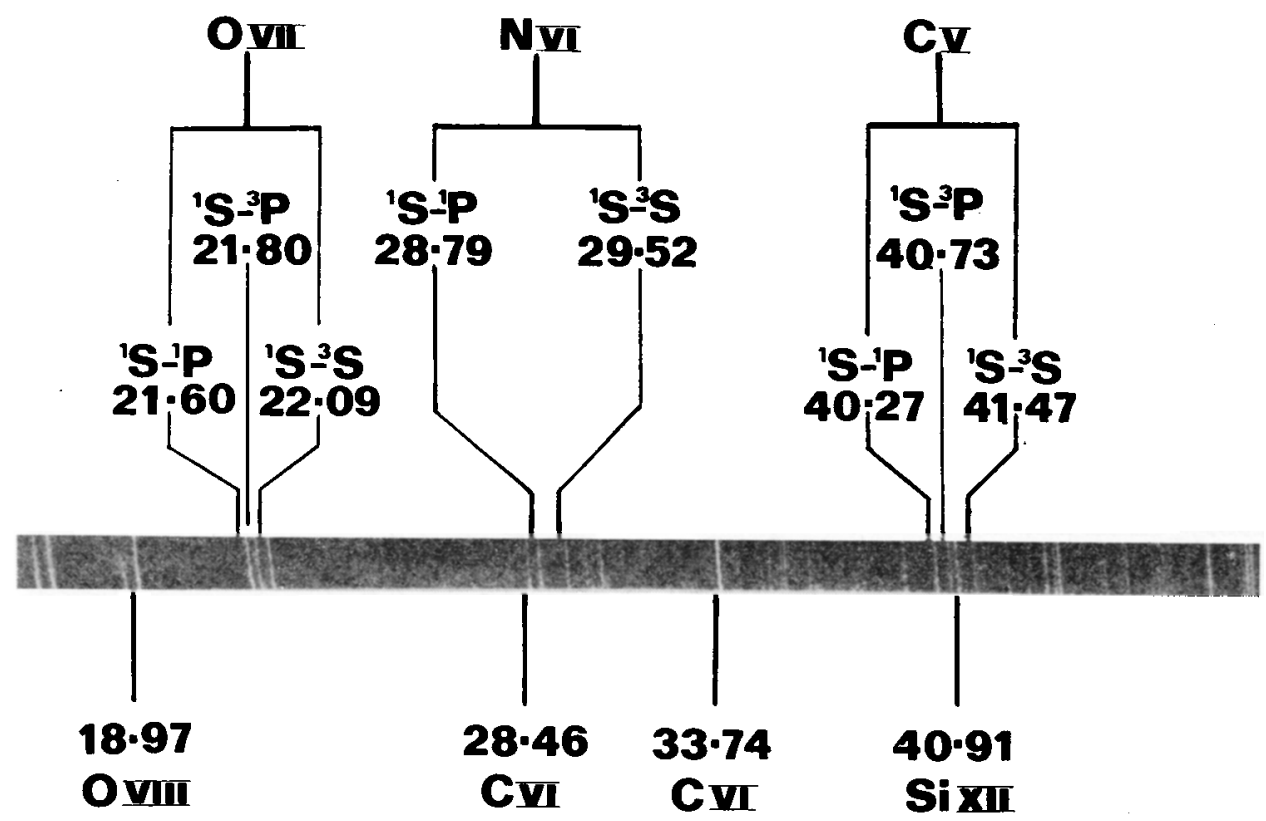

Fig. 6. A spectrum photographed at grazing incidence by the Culham Laboratory on 20 November 1969. The helium-like triplets of $\mathrm{CV}, \mathrm{N} \mathrm{vI}_{\mathrm{I}}$ and $\mathrm{O} \mathrm{vII}_{\mathrm{I}}$ are shown with excellent resolution (Freeman and Jones, 1970).

transition ${ }^{1} P-{ }^{1} S$, the intercombination ${ }^{3} P$ to ${ }^{1} S$, and the forbidden ${ }^{3} S-{ }^{1} S$. For $\mathrm{N}$ vil the intercombination line was apparently too weak to record. In the Bragg spectrometer range the triplets of NeIX, Nax, MgXI, AlxII, SixIII, Sxv, ArxviI, CaxIX, and Fexxv have now been observed from active regions or flares. (See Neupert and Swartz, 1970; Doschek and Meekins, 1970; Walker and Rugge, 1970.) As Gabriel and Jordan (1969) have shown, the intensity ratio of the forbidden to the intercombination line provides data from which the electron density in the source can be determined with high sensitivity. 
To learn more about the Sun's atmosphere, particularly the chromosphere and corona, and about solar phenomena in general, it is close to necessary to observe with the greatest possible spatial resolution, and to make use of the resonance lines of highstage ions which lie in the XUV. Therefore XUV spectroheliograms of high spatial resolution must be obtained in many emission lines covering different parts of the chromosphere, transition region, and corona. Considerable progress has been made in this direction in the last few years.

Spectroheliograms in the $\mathrm{Mg}_{I I} \mathrm{H}$ and $\mathrm{K}$ lines near $2800 \AA$ are of great theoretical interest because of their close relation to $\mathrm{CaII} H$ and $\mathrm{K}$, and the probable higher position in the chromosphere from which the emission cores of $\mathrm{MgII}$ are emitted than for CaII. H Ly- $\alpha$ spectro-heliograms should be recorded together with $\mathrm{Mg}$ II and $\mathrm{Ca} I \mathrm{H}$ and $\mathrm{K}$, because they map a still higher part of the chromosphere. Off-band $\mathrm{H}$ Ly- $\alpha$ spectroheliograms are also needed for the full interpretation, because Ca II and $\mathrm{Mg} I \mathrm{l}$ are ionized by the $\mathrm{H} \mathrm{Ly}-\alpha$ radiation that is present in these regions of the chromosphere, and its radiation density is unknown, as Athay and Zirker (1962) have pointed out.

A start in this direction has been made by Fredga (1969), who obtained spectroheliograms in MgII, 2795.5 $\AA$ on May 20, 1968, and 2802.7 $\AA$ in 1965 and 1966. Use was made of a Šolc filter, flown in Aerobee- 150 rockets. Results of the 1968 flight are reproduced in Figure 7. This filter was a double Šolc with a FWHM bandpass of $2.1 \AA$. The $\mathrm{Mg}$ II, $\mathrm{K}$-line image is compared with images in CaII $\mathrm{K}, \mathrm{H} \alpha$, and soft $\mathrm{X}$ rays. The spatial resolution is of the order of $10 \mathrm{arc} \mathrm{sec}$. Qualitatively, the $\mathrm{Mg}$ II and Ca II images are similar, showing the same plages, and having an indication of supergranular cells that are much the same. In the H Ly- $\alpha$ spectroheliogram of 1959 (Purcell et al., 1959) which had 20-30 arc sec spatial resolution, the contrast between plages and quiet regions was double that in $\mathrm{Ca}$ II $\mathrm{K}$; hence, a somewhat enhanced contrast might be expected in $\mathrm{Mg}$ II. Actually, the contrasts in the $\mathrm{Mg}$ and $\mathrm{Ca}$ images were found to be similar, but this may be attributed to the much wider bandpass for MgII than for Ca II K (0.1 $\AA)$.

The emergence and development of plages with increasing altitude in the solar atmosphere can now be traced, at least qualitatively, by comparing spectroheliograms and stigmatic spectra as recorded in lines excited at different temperatures. In white light from the deep photosphere the plages show hardly at all except faintly near the limb. In strong Fraunhofer lines they are brighter, and in the chromospheric core of $\mathrm{Ca} I \mathrm{~K}$ and $\mathrm{Mg}$ II $\mathrm{K}$ they are still brighter relative to the photospheric background. A similar change is observed in the ultraviolet. In Figure 8 , an image in the band 2200-2275 $\AA$, photographed from a balloon on October 3, 1966 (Bonnet and Blamont, 1968 ) is seen to be similar to a white light image in that it shows only suggestions of plages close to the limb. In the band 1940-2020 $\AA$, however, the plages are clearly present and resemble CaIIK. In the ranges 1100-2000 $\AA$ and 1800-2076 $\AA$ stigmatic spectra show the presence of plages whose contrast generally increases as the wavelength is decreased (Bonnet et al., 1967; Tousey, 1967; Tousey et al., 1970). Thus the plages first become clearly present in the region $i<2076 \AA$ where radiation from the 

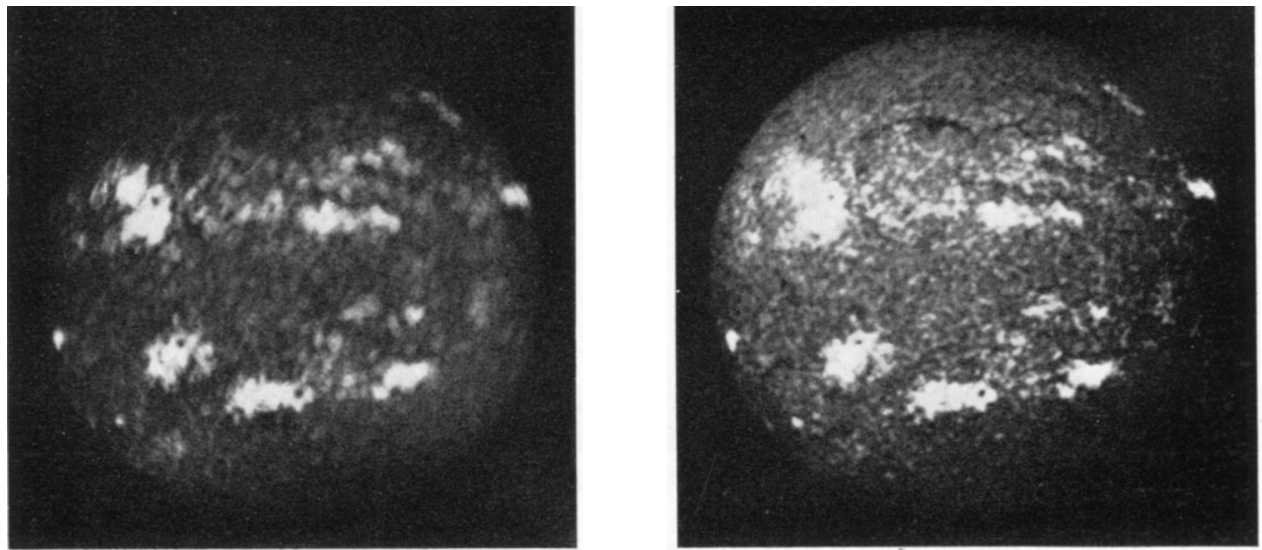

\section{GSFC}

Mg II U.T. 15.03

Stockholm Obs.

CaII U.T. 15.15
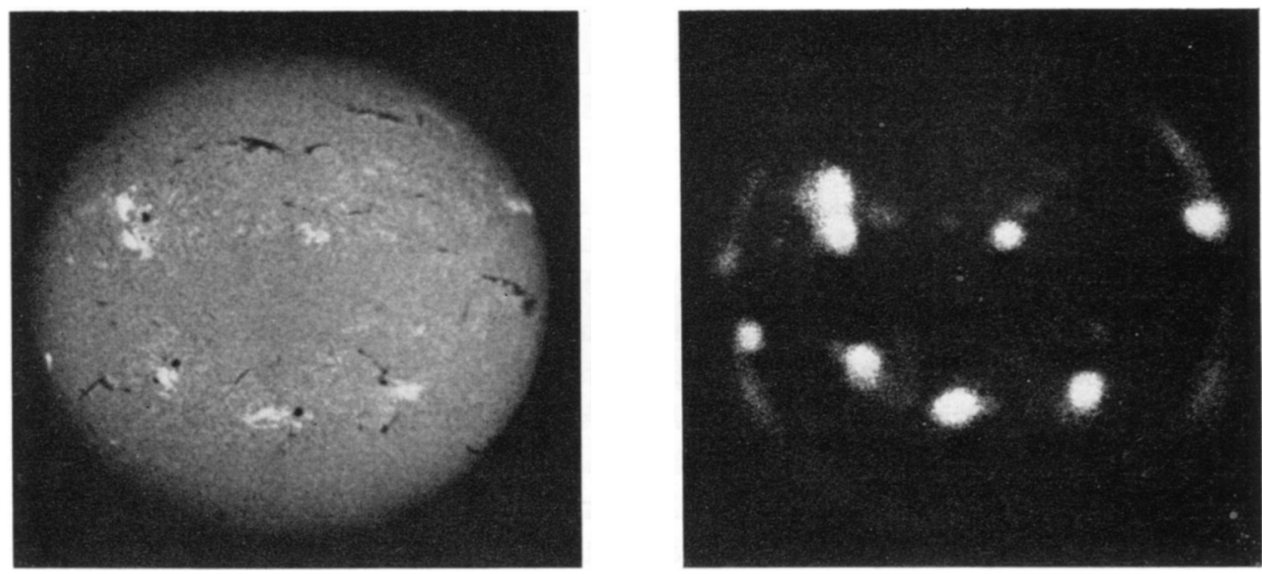

\section{Ha U.T. 15.03}

20 MAY 1968

$$
\lambda=44-70 \AA
$$

Fig. 7. Monochromatic images obtained from a rocket flown by the Goddard Space Flight Center on 20 May 1968 (Fredga, 1969). Upper left; MgII K line, $2795.5 \AA$; lower right, X-ray filter heliogram, $\lambda<33 \AA$ plus $44-70 \AA$ (Fredga, Muney and Underwood). $\mathrm{H} \alpha$ from Anacapri and $\mathrm{Ca} \mathrm{K}$ from McMath Hulbert.

temperature minimum begins to predominate; as the wavelength becomes shorter the height of formation where they radiate increases, and their contrast relative to the continuum becomes greater; when the chromosphere predominates in the $\mathrm{SiI}^{3} P$ recombination continuum below $1530 \AA$, a further enhancement of plage to continuum contrast is observed.

The emission line of greatest interest is probably H Ly- $\alpha 1216 \AA$. Curiously, the 1959 NRL image (Purcell et al., 1959) is still the highest resolved image obtained to the present time. It shows detail to $20-30 \mathrm{arc} \mathrm{sec}$. but there is no evidence of limb 


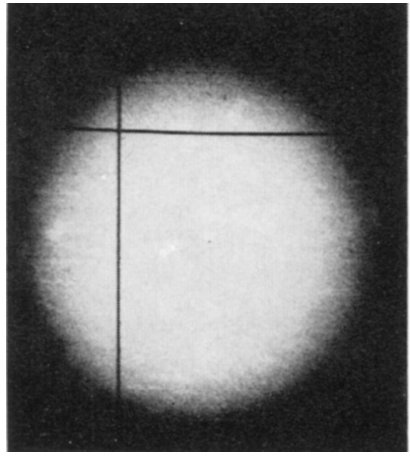

$2235 \pm 40 \AA$

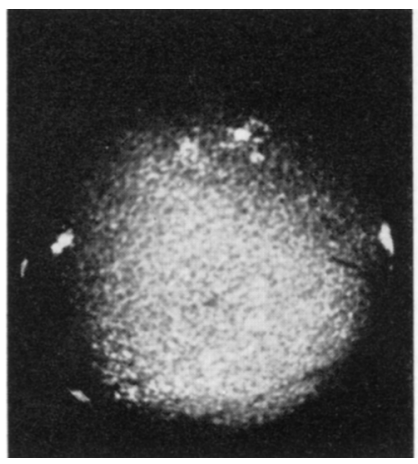

Cok

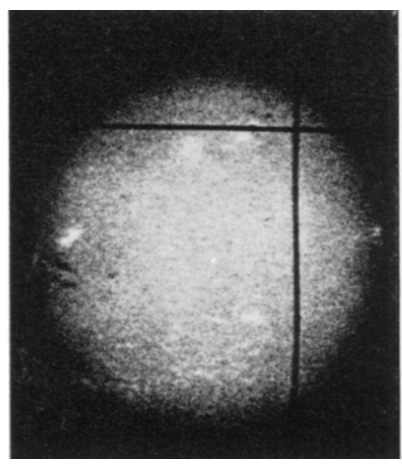

$1940 \pm 40 \AA$

Fig. 8. UV solar images from a high altitude balloon on 3 October 1966 made at the shortest wavelength end of the normal photosphere (2235 $\AA$ ) and near the temperature minimum $(1940 \AA)$, (Bonnet and Blamont, 1968), compared with chromospheric Ca II K.

brightening or darkening. Recently, however, there have been two new types of experiment that record spatial structure in H Ly- $\alpha$. Sloan (1968) of NRL, using a telescope with a 2 arc sec field of view and an ion chamber sensitive mainly to Ly- $\alpha$, observed fluctuations in output as the biaxial pointing control system made corrections in pointing. He concluded that the quiet sun in $\mathrm{H}$ Ly- $\alpha$ shows detail to at least 2 arc sec and with contrast approaching 2 . A different method was applied by Blamont and Malique (1969) who flew a photoelectric photometer into the total eclipse shadow on 12 November 1966. After correction for a plage, they report $30 \%$ limb brightening for $\mathrm{H} \mathrm{Ly- \alpha}$ in a ring 15 arc sec wide and centered 6.4 arc sec inside the infrared limb. So narrow and small an enhancement would not have been detected in the NRL 1959 image, especially since this was photographed at solar maximum and the image was covered with plages. A H Ly- $\alpha$ image with 1 arc sec spatial resolution would be of great interest.

By far the most extensive series of XUV spectroheliograms has been made with spectroheliographs installed in OSO 5 by NRL and in OSO 4 and 6 by the Harvard College Observatory. In this paper I can do no more than touch on the results that are pouring in, especially from OSO 6 . The spatial resolution is limited to 1 arc min in OSO 4 and 5. In OSO 6 the resolution is $35 \times 35$ arc sec; a spectroheliogram can be built up for the entire Sun in $8 \mathrm{~min}$, or for a $7.5 \times 7$ arc min area, chosen at any position, once every $30 \mathrm{~s}$.

Figure 9 shows a sampling of five of Harvard's spectroheliograms from OSO 4 (Goldberg, 1969), compared with the Fraunhofer Institut map and a plot of the FexIV intensity for that day. The images are arranged in order of increasing temperature: the Lyman continuum, $10^{5} \mathrm{~K}$, showing plages and generally similar to $\mathrm{H}-\alpha$; $\mathrm{N}$ III, $10^{5} \mathrm{~K}$, with more contrast and a little limb brightening; OVI, $3.25 \times 10^{5} \mathrm{~K}$ with still more contrast, stronger limb brightening, and some emission in the corona beyond the limb; $\mathrm{Mgx}, 1.4 \times 10^{6} \mathrm{~K}$, from the transition region and corona, showing much emission beyond the limb: Sixir, at more than $2 \times 10^{6} \mathrm{~K}$, extending well into the 


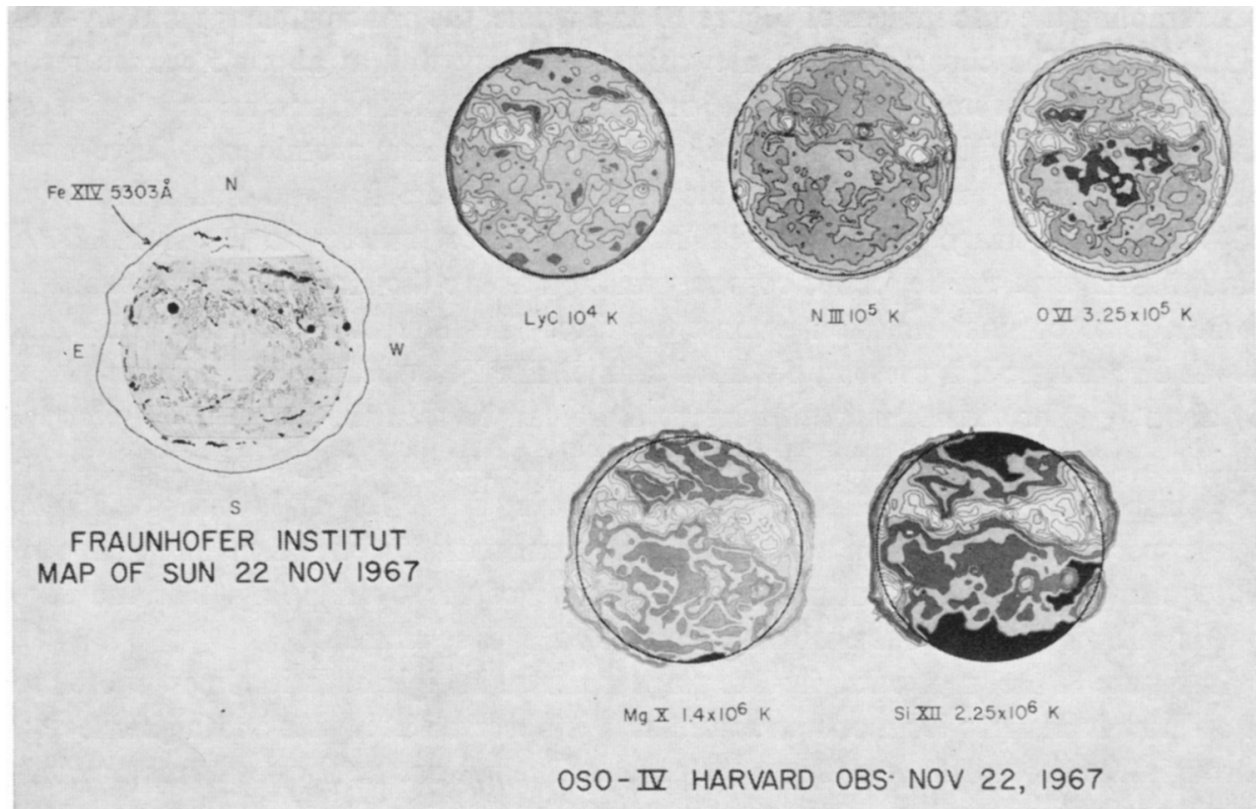

Fig. 9. Spectroheliograms of $1 \times 1$ arc min resolution made by the Harvard College Observatory photoelectric spectrometer on OSO 4. The images are selected to illustrate the changing pattern of emission in passing upward through the chromosphere and into the corona. The ground-based solar data for the same day are presented for comparison from the map published by the Fraunhofer Institut.

corona and showing emission mainly from the corona and plages. The excellence of the correlation between $\mathrm{MgX}$ and FexIV shows that these ions reach maximum emission intensity at about the same temperature, in accordance with ionization theory. Many thousands of these spectroheliograms have been recorded and will soon appear in Atlas form (Reeves and Parkinson, 1970).

The XUV spectroheliograms having the greatest spatial resolution, however, are those obtained by photography from rockets (Austin et al., 1967; Tousey et al., 1968). The technique is simple; a single concave grating is used in a Wadsworth configuration with a filter of thin aluminum placed in front of the film to eliminate long wavelength stray light. Figure 10 is a section of perhaps the best obtained as yet, not only because it has the greatest spatial resolution, approaching 3 arc sec in short exposures, but also because it shows the morphological character of a flare in many XUV emission lines. The instrument produces monochromatic solar images, spread out along the direction of dispersion. Through the use of a $2400 \mathrm{l} / \mathrm{mm}, 2 \mathrm{M}$ radius grating, there was produced a spectrum of solar images $9.5 \mathrm{~mm}$ in diameter, each spanning $39 \AA$ at a linear dispersion of $4.1 \AA / \mathrm{mm}$. Because the images overlap, the full analysis to derive intensities requires deconvolution. However, many features are quite well separated. The flare is the string of tiny dots between arrows. At the bottom is noted the location of the flare in several important emission lines. 
Examining the disc images of Figure 10 as a whole, the principal image is $\mathrm{H} \mathrm{Ly}-\alpha$ of HeII, $304 \AA$. The chromospheric network shows very well at about 5 arc sec resolution in this exposure. On some of the original images, spicules, or clumps of spicules, are easily distinguished. Often in the past great quiescent prominences have been observed in HeII, but on this date the prominences were few and small. In Fexv, $284 \AA$, the disc image is located with its center almost exactly on the He II, $304 \AA$ limb, but all that can be seen is coronal emission above the limb, active regions, and some diffuse coronal emission against the disc. The flare in Fexv falls within the $304 \AA$ disc image; it is present, but hard to find. The FexVI, $335 \AA$ disc overlaps HeII by about $\frac{1}{4}$ solar diameter; in this image there can be seen the flare, strong coronal emission over the nearby limb, and plages, the principal one being the great butterflylike plage in the lower left. The section of sharp ring at the very right is MgIX, $369 \AA$. These spectroheliograms contain a tremendous amount of information, but great care is required to extract quantitative data because of the overlapping. For the flare overlapping is not a serious problem because its area is so small.

In Figure 11 the great butterfly-like plage is shown in eight emission lines, chosen to show how it changes form and structure as it is observed at higher and higher levels, through the chromosphere and into the corona. The cut-out section measures approxi-

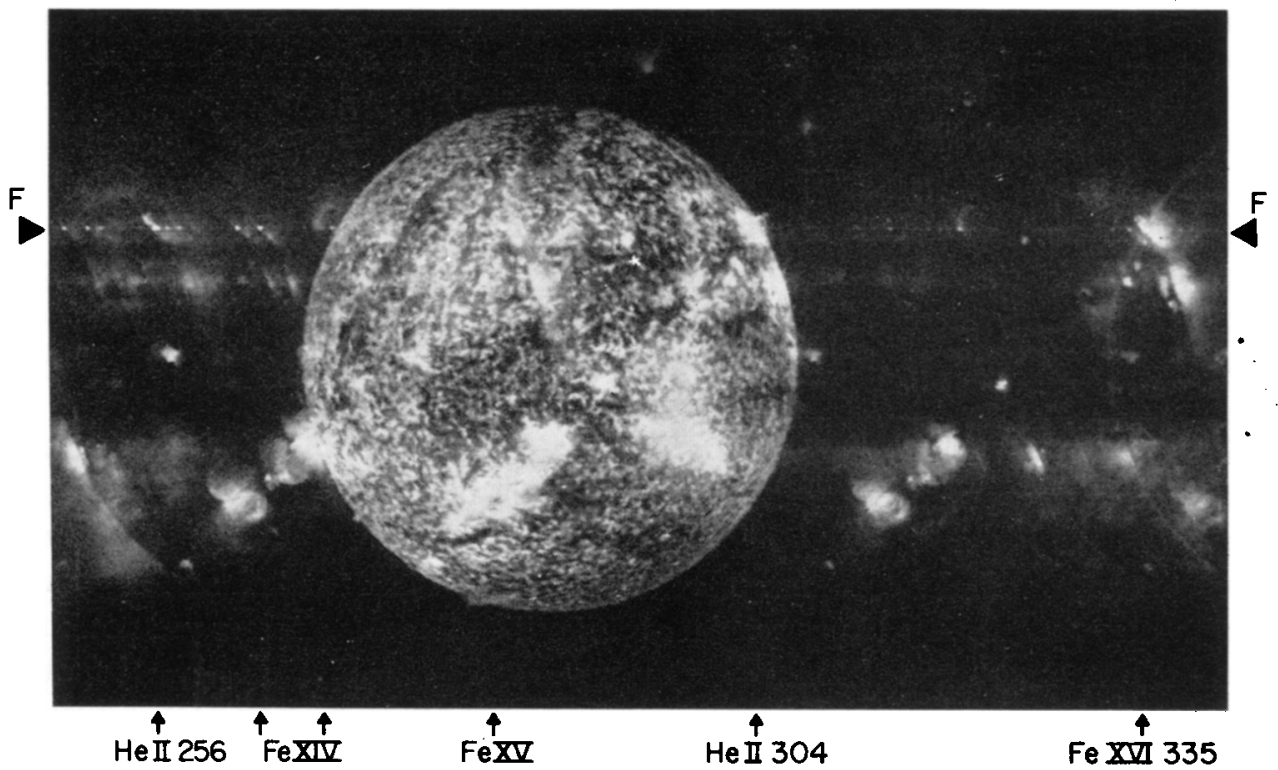

FLARE OF IMP. 24 NOV. 1969 20:33 UT

Fig. 10. Part of an XUV spectroheliogram obtained by NRL from an Aerobee rocket launched during an importance $2 \mathrm{~N}$ flare on 4 November 1969. The flare nucleus is present in many emission lines as a string of dots between the arrows F. In HeII $256 \AA$ and $304 \AA$ the flare nucleus is accompanied by threadlike emission. The resolution is $5 \mathrm{arcsec}$, shows clearly in He II $304 \AA$ the chromospheric network, a few quiet prominences, and on the original, spicules or clumps of spicules. The butterfly-like plage is perhaps the most spectacular feature present. 


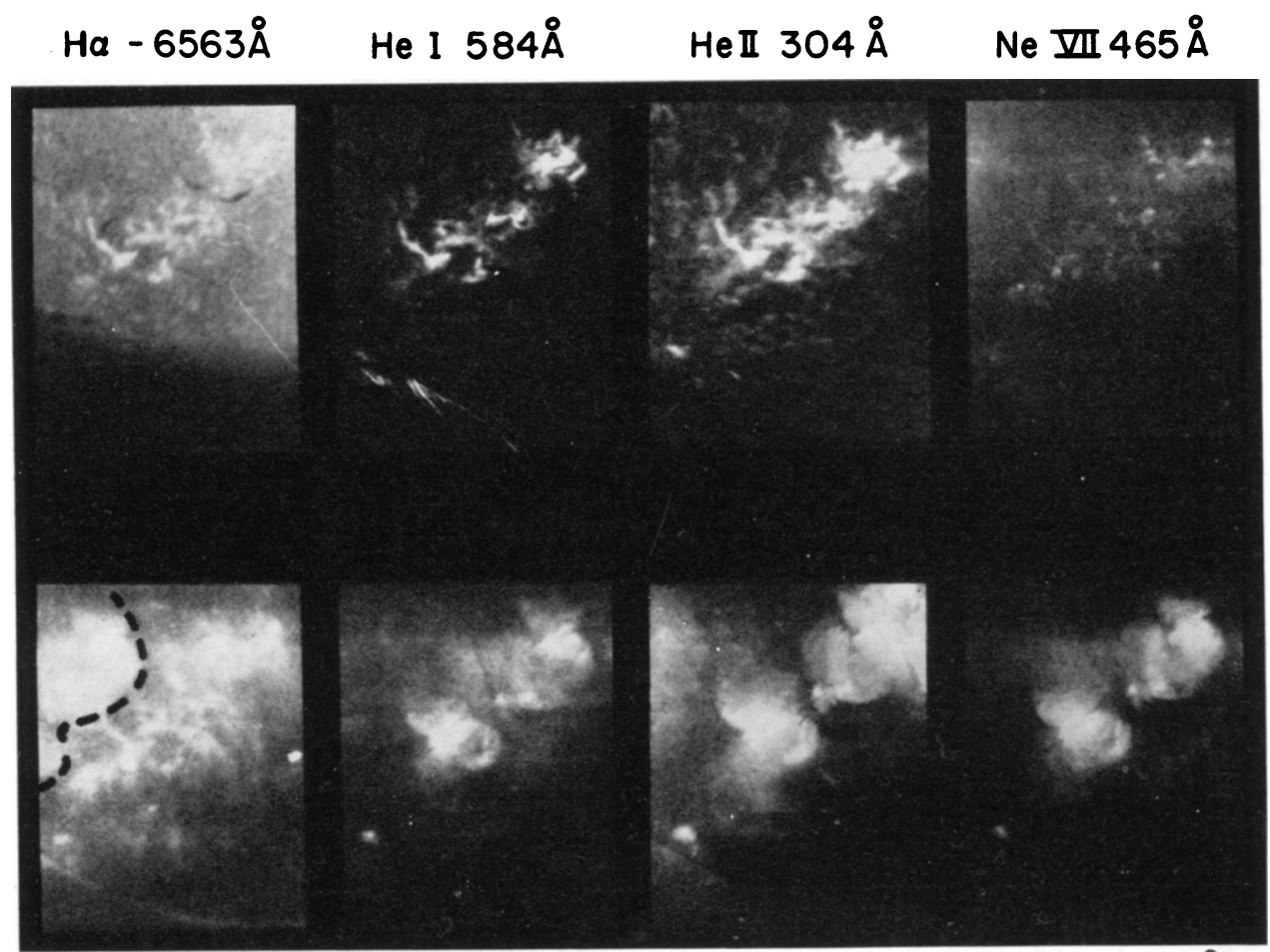

Mg X $368 \AA \quad$ Si XI $499 \AA \quad$ Fe XV $284 \AA \quad$ Fe XVI $335 \AA$

\section{FDVU-A2- LAUNCH 4 NOV 69}

Fig. 11. The butterfly-like plage in 8 lines ascending in altitude through the chromosphere and corona. The $\mathrm{H}_{\mathrm{x}}$ image is from ESSA, Boulder, Colo. In MgIx the section to the left of the dotted line is produced by Fexvi $361 \AA$.

mately $4 \times 5$ arc min. The $\mathrm{H}-\alpha$ image resembles HeI $584 \AA$ and He II $304 \AA$, but in the latter two the background is less intense, dark filaments are broader, the chromospheric network is more pronounced. The next image, Ne VII, $465 \AA$, originates at about $500000 \mathrm{~K}$ in the uppermost part of the chromosphere; the emission is diffuse in character; dark filaments and the chromospheric network are all but gone as is characteristic of all Ne viII images recorded up to now. Some of the diffuse emission is probably coronal. A trace of the narrow, uniform bright limb, characteristic of this line, is visible in the reproduction; clearly, the emission layer is optically thin, except at the limb and in plages. Continuing higher, in MgIX, maximizing at $10^{6} \mathrm{~K}$ and lying in the transition region the detailed structure of the plage has nearly disappeared and has been replaced by diffuse, intense localized emission that is beginning to take on a new form. The activity beyond the dotted line belongs to Fexvi, $361 \AA$. In Si XII, $499 \AA$. a $2 \times 10^{6} \mathrm{~K}$ truly coronal line, the butterfly form first becomes apparent, and the chromospheric details have disappeared. No limb ring is present. Some of the diffuse 
background is from the He I continuum whose limit lies at $504 \AA$. In Fexv, $284 \AA$, and FexvI, $335 \AA$, both close to $3 \times 10^{6} \mathrm{~K}$, the plage has fully developed its coronal form, which is not at all like its form in the chromosphere. There seem to be some small differences between FeXV and XVI, but nearby faint lines may contribute and must be taken into account. Coronal emission above the dark disc is seen in Fexv at the lower left corner. When such a plage can be followed in orderly steps from the outer photosphere or temperature minimum where it first appears, across the minimum, up through the chromosphere and corona, it will be possible to derive the electron density and electron temperature distribution in the plage in three dimensions and to learn much more about its origin and stability.

The flare itself is shown in Figure 12 in 13 emission lines arranged as in Figure 11 by increasing temperature. In $\mathrm{H} \alpha$ the flare was classified as of importance $2 \mathrm{~N}$. The XUV images were obtained $5 \mathrm{~min}$ after $\mathrm{H} \alpha$ maximum, in the period when the flare was dying. From a rocket instrumented by the American Science and Engineering Corporation (Krieger et al., 1970) and flown just before the NRL rocket, X-ray images of the same flare overlapping the NRL series by about one minute were photographed with a Wolter lens telescope and various filters.

$\mathrm{H} \alpha$, at the upper left, is shown near $\mathrm{H} \alpha$ maximum, in a photograph from ESSA, Boulder, Colorado. The flare nucleus lies near the center of the long plage that is enhanced, especially above the nucleus. In HeI, $584 \AA$, and HeII, $304 \AA$, the flare is much brighter and larger than in $\mathrm{H} \alpha$, but it has roughly the same form. The detail is different, but $\mathrm{H} \alpha$ was obtained 5 min earlier. The image of OIV is confused because

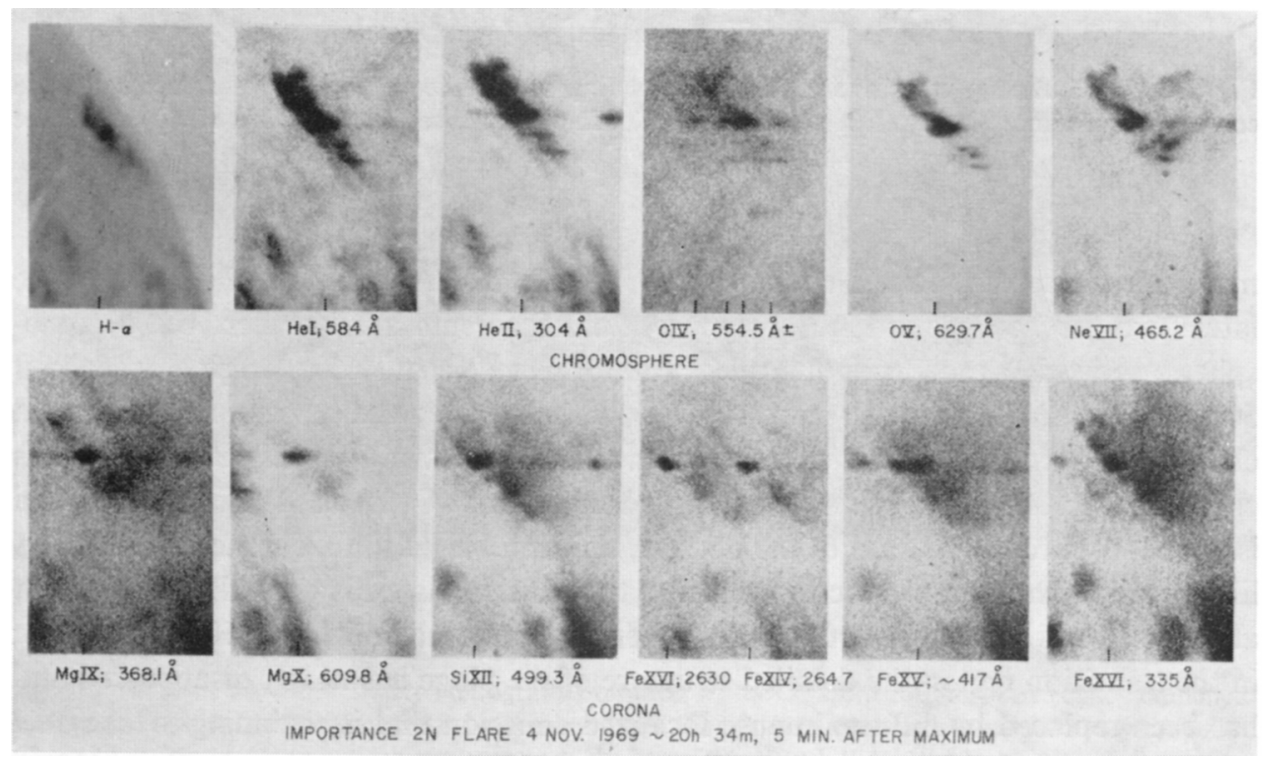

Fig. 12. The 4 November 1969 importance $2 \mathrm{~N}$ flare in 13 lines, ascending through the chromosphere and corona. $\mathrm{H} \alpha$ is from ESSA, Boulder, Colo. The magnification and position of the limb is the same in each image (Naval Research Laboratory). 
this is a multiplet with four components. In Ov and Ne vII, maximizing at 200000 and $500000 \mathrm{~K}$ respectively, the nucleus is intense, and the rest of the flare structure is very complicated, especially so in Ne VII. There is threadlike emission above the nucleus that is certainly associated with the flare. Below the nucleus is a pattern of small emission nuclei; it is not the same for the two lines and seems to be associated with the flare. The first signs of coronal emission above the limb appear in Ne VII. MgIX, which maximizes at $10^{6} \mathrm{~K}$ shows the nucleus but with a great deal more coronal emission nearby and over the limb; the detailed emission in the plage has become reduced or diffused. $\mathrm{Mgx}$ is similar, but the exposure is much weaker, hence the coronal emission appears less prominent. In SiXII, $2 \times 10^{6} \mathrm{~K}$, the emission in the corona is somewhat increased. This trend continues to FexvI, the highest excitation ion recorded. Here the nucleus is still small and intense and in the same position, the coronal cloud has increased in intensity, the bright filament is absent, but is replaced by spotty diffuse emission.

The area covered by the $\mathrm{H} x$ flare was reported as 2.1 square degrees apparent at maximum. In HeII, 2 min after maximum, the total area was 6 square deg, 3 times greater than $\mathrm{H} x$ assuming that it was measured in the same way. In the coronal lines, disregarding the coronal cloud, the area of the flare nucleus was 0.1 square deg, derived from its apparent diameter, estimated as 6 arc sec after correcting for pointing jitter, and instrumental profile.

To obtain flare spectra such as these, or to make other observations of flares from rockets is extremely difficult because of launch constraints imposed by range schedules. Orbiting vehicles overcome this problem. In the Apollo Telescope Mount project, that now forms a part of Skylab, which is planned to be the first large manned space station, the NRL hopes to obtain XUV spectroheliograms and flare images like these, but with twice the magnification, and to do so from the very beginning of the flare. The astronaut will be on duty, waiting for a flare to appear; when it does, he will set the instrument into an automatically programmed sequence of exposures. At present this is scheduled to take place in late 1972 and 1973, far from solar maximum, but still not at minimum. Some luck is required, but we believe that in ATM we shall be successful obtaining greatly improved images of a flare in many XUV emission lines, and shall observe the development of at least one flare from beginning to end.

\section{References}

Athay, R. G. and Zirker, J. B.: 1962, Astrophys. J. 136, 242.

Austin, W. E., Purcell, J. D., Snider, C. B., Tousey, R., and Widing, K. G.: 1967, in Smith-Rose (ed.), Space Res. 7, 1252, North-Holland Publ. Co., Amsterdam.

Bates, B., Bradley, D. J., McKeith, C. D., McKeith, N. E., Burton, W. M., Paxton, H. J. B., Shenton, D. B., and Wilson, R.: 1969, Nature 224, 161.

Blamont, J. E. and Malique, C.: 1969, Astron. Astrophys. 3, 135.

Boland, B. C., Jones, B. B., Wilson, R., Engstrom, S. R. T., and Noci, G.: 1970 (to be published).

Bonnet, R. M.: 1968, Ann. Astrophys. 31, 597.

Bonnet, R. M. and Blamont, J. E.: 1968, Solar Phys. 3, 64.

Bonnet, R. M. Blamont, J. E., and Gildwarg, P.: 1967, Astrophy:s. J. 148, L1 15.

Brueckner, G. E., Bartoe, J. F., Nicolas, K. R., and Tousey, R.: 1970, Nature 226, 1132. 
Burton, W. M. and Wilson, R.: 1965, Nature 207, 61.

Doschek, G. A. and Meekins, J. F.: 1970, Solar Phys. 13, 220.

Fredga, K.: 1969, Solar Phys. 9, 358.

Freeman, F. F., and Jones, B. B.: 1970, Solar Phys. 15, 288.

Freeman, F. F., Gabriel, A. H., Jones, B. B., and Jordan, C.: 1970 (to be published by the Royal Society).

Gabriel, A. H. and Jordan, C.: Monthly Notices Roy. Astron. Soc. 145, 241.

Goldberg, L.: 1969, Sci. Am. 220, 92.

Hinteregger, H. E. and Hall, L. A.: 1969, Solar Phys. 3, 175.

Jones, B. B., Boland, W. C., Wilson, R., and Engstrom, S. T. F.: 1970, in L. Houziaux and H. E. Butler (eds.), 'Ultraviolet Stellar Spectra and Related Ground-Based Observations', IAU Symp. 36, 271-3, D. Reidel, Dordrecht, Holland,

Krieger, A., Vaiana, G., Van Speybroeck, L., Zehnpfennig, T., and Zombeck, M.: 1970, Bull. Am. Phys. Soc. 15, 612.

Lemaire, P.: 1969, Astrophys. Letters 3, 43.

Neupert, W. M. and Swartz, M.: 1970, Astrophys. J. Letters 160, L189.

Neupert, W. M., White, W. A., Gates, W. J., Swartz, M., and Young, R. M.: 1969, Solar Phys. 3, 183.

Parkinson, W. H. and Reeves, E. M.: 1969, Solar Phys. 10, 342.

Purcell, J. D., Packer, D. M., and Tousey, R.: 1959, Nature 184, 8.

Reeves, E. M. and Parkinson, W. H.: 1970, Astrophys. J. Suppl. 21, No. 181,

Sloan, W. A.: 1968, Solar Phys. 4, 196 and 5, 329.

Speer, R. J., Garton, W. R. S., Morgan, J. F., Nicholls, R. W., Goldberg, L., Parkinson, W. H., Reeves, E. M., Jones, T. J. L., Paxton, H. J. B., Shenton, D. B., and Wilson, R.: 1970, Nature 226, 249.

Tousey, R.: 1964, J. Roy. Astron. Soc. 5, 123.

Tousey, R.: 1967a, Appl. Opt. 6, 2044.

Tousey, R.: 1967b, Astrophys. J. 149, 239.

Tousey, R., Purcell, J. D., Austin, W. E., Garrett, D. L., and Widing, K. G.: 1964, in P. Muller (ed.) Space Res. 4, 703, North-Holland Publ. Co., Amsterdam.

Tousey, R., Purcell, J. D., and Garrett, D. L.: 1967, Appl. Opt. 6, 365.

Tousey, R., Purcell, J. D., Garrett, D. L., and Widing, K. G.: 1970 (unpublished).

Tousey, R., Sandlin, G. D., and Purcell, J. D.: 1968, in K.O. Kiepenheuer (ed.), 'Structure and Development of Solar Active Regions', IAU Symp. 35, 411.

Walker, A. B. C. and Rugge, H. R.: 1970, Astron. Astrophys. 5, 4.

Widing, K. G., Purcell, J. D., and Sandlin, G. D.: 1970, Solar Phys. 12, 52.

REFERENCES TO SOLAR CONSTANT:

Allen, C. W.: 1958, Astrophysical Quantities, 2nd ed., p. 168, University of London, 1963.

Arveson, J. C., Griffin, R. N., Jr., and Pearson, B. D., Jr.: 1969, Appl. Opt. 8, 2215.

Drummond, A. J., Hickey, J. R., Scholes, W. J., and Laue, E. G.: 1968, Nature 218, 259.

Johnson, F. S.: 1954, J. Meteorol. 11, 431.

Kondratiev, K. Ya, Nikolsky, G. A., Badinov, I. Ya, and Andreev, S. D.: 1967, Appl. Opt. 6, 197.

Labs, D. and Neckel, H.: 1968, Z. Astrophys. 69, 1.

Makarova, E. A. and Kharitonov, A. V.: 1968, Astron. Zh. 45, 752.

Murcray, D. G., Kyle, T. G., Kosters, J. J., and Gast, P. R.: 1968, The Measurement of the Solar Constant from High Altitude Balloons, University of Denver, Colo.

Stair, R. and Ellis, H. T.: 1968, J. Applied Meteorol. 7, 635.

Stair, R. and Johnston, R. G.: 1956, J. Nat. Bureau Standards 57, 205.

Thekaekara, M. P., Kruger, R., and Duncan, C. H.: 1969, Appl. Opt. 8, 1713. 